\title{
Computergestützte chirurgische 3D-Planung und schablonengeführte Implantation
}

Schneider, David

DOI: https://doi.org/10.1024/1661-8157/a002526

Posted at the Zurich Open Repository and Archive, University of Zurich ZORA URL: https://doi.org/10.5167/uzh-139618

Journal Article

Accepted Version

Originally published at:

Schneider, David (2016). Computergestützte chirurgische 3D-Planung und schablonengeführte Implantation. Praxis, 105(23):1381-1387.

DOI: https://doi.org/10.1024/1661-8157/a002526 
Computer-unterstützte chirurgische 3D-Planung und schablonengeführte Implantation Computer-assisted Surgical 3D Implant Planning and Template-guided Implant Placement

David Schneider, Dr. med., Dr. med. dent.

\section{Oberarzt}

Universität Zürich, Zentrum für Zahnmedizin

Klinik für Kronen- und Brückenprothetik, Teilprothetik und zahnärztliche Materialkunde

Plattenstrasse 11, 8032 Zürich

+41446343258 Telefon; +41446344305 Telefax

www.dent.uzh.ch

david.schneider@zzm.uzh.ch

Privatpraxis

Praxis Grunder und Schneider

Dufourstrasse 7a, 8702 Zollikon

+41443915488 Telefon; +41443918920 Telefax

www.zahnaerzte-zollikon.ch

info@zahnaerzte-zollikon.ch 


\section{Im Artikel verwendete Abkürzungen}

- CAD: Computer Aided Design, Computer-unterstützte Gestaltung

- CAIPP: Computer-Assisted Implant Planning and Placement, Computer-assistierte Implantatplanung und schablonen-geführte Implantatinsertion

- CAM: Computer Aided Manufacturing, Computer-unterstützte Herstellung

- CBCT: Cone Beam Computer Tomography, Digitale Volumentomographie

- CT: Computertomographie

- DVT: Digitale Volumentomographie

- GBR: Guided Bone Regeneration, Geführte Knochenregeneration

- OPT: Orthopantomogramm

- Rx: Röntgen

\section{Schlüsselwörter}

- Zahnimplantate, Computer-assistierte Implantologie, Schablonen-geführte Chirurgie, Digitale Zahnmedizin

- Dental implants, Computer-assisted implantology, Template-guided surgery, Digital dentistry

- Implants dentaires, Implantologie assistée par ordinateur, chirurgie guidée, dentisterie numérique

\section{Zusammenfassung}

Dentale Implantate werden mit hohen Erfolgsraten zur Verankerung von Prothese, Brücken und Kronen eingesetzt. Der Erfolg der Behandlung hängt jedoch unter anderem von einer korrekten Position des Implantates ab, Fehlpositionen können zu biologischen, funktionellen und ästhetischen Komplikationen führen. Die bisherige, konventionelle Planung einer implantatprothetischen Behandlung erfolgt mit Hilfe von konventionellen Röntgenbildern und die Implantate werden nach freihändiger Osteotomie eingesetzt. Der zunehmende Einsatz der digitalen Volumentomographie in der Zahnmedizin und die Weiterentwicklung von reiner Bildbetrachtungszur technisch fortgeschrittenen Implantationsplanungs-Software ermöglichen heute dem Kliniker eine umfassende, dreidimensionale präoperative Analyse und chirurgische Vorbereitung. Durch CAD-CAM-Technologie kann die geplante Implantatposition in eine Bohrschablone übertragen werden, die intraoperativ zu Führung der Instrumente eingesetzt wird, was in vielen Fällen die Implantation erleichtert. 


\begin{abstract}
Dental implants are succesfully used for the retention of removable dentures, fixed dental prostheses and single crowns. The success of the treatment, however, significantly depends on a correct implant position. Malplaced implants can cause biological, functional and esthetic complications. So far, conventional planning of an implant-prosthetic treatment includes conventional x-rays and the implants are placed after free-hand osteotomy. The growing use of cone beam computer tomography in dentistry and the further development of the viewing software towards a sophisticated implant planning software allow the clinician a comprehensive 3D preoperative analysis and virtual surgical preparation. By CAD-CAM technology the planned implant position can be transferred into a surgical guide that is used for instrument guidance, which often simplifies the implantation procedure.
\end{abstract}

\title{
Résumé
}

Les implants dentaires sont utilisés avec grand succès pour l'ancrage des prothèses, des ponts et des couronnes. Cependant, le succès du traitement dépend entre autres de la position correcte de l'implant. Les positions incorrectes peuvent causer des complications biologiques, fonctionnelles et esthétiques. La planification classique d'un traitement implanto-prothétique est effectuée en utilisant des radiographies conventionnels et les implants sont insérés après ostéotomie à main levée. L'utilisation croissante de la tomographie à faisceau conique en dentisterie et le développement de la software d' une visualisation d'images pur pour un logiciel de planification de l'implant techniquement avancé permettent maintenant le clinicien, une analyse pré-opératoire complète en trois dimensions et une préparation chirurgicale amélioré. Grâce à la technologie CAO-FAO, la position de l'implant prévue peut être transféré dans un guide qui est utilisé pendant l'opération pour guider des instruments, ce qui souvant facilite l'implantation. 


\section{Einleitung}

Die Einführung dentaler Implantate in der Zahnmedizin hat die therapeutischen Möglichkeiten massgebend und nachhaltig verändert. Dank im Kieferknochen verankerter schraubenförmiger Zahnimplantate können zahnlose Patienten, statt mit rein auf der Schleimhaut aufliegenden und häufig schlecht sitzenden Prothesen, nunmehr mittels implantatgetragenen festen Brücken oder druckknopfverankerten Prothesen versorgt werden. Effizientere Nahrungszerkleinerung, positiver Einfluss auf die Ernährung, mehr Sicherheit und Komfort sowie Selbstvertrauen bei sozialer Aktivität sind einige Vorteile, die eine stabilere prothetische Versorgung bietet [1]. Bei teilbezahnten Patienten, die einzelne Zähne durch Trauma, Karies oder Parodontitis verloren haben, bieten Implantate die Möglichkeit, festsitzenden, ästhetisch und funktionell hochwertigen Zahnersatz durch implantatbefestigte Kronen oder Brücken zu bewerkstelligen. Das invasive und irreversible Beschleifen von Nachbarzähnen für Brückenlösungen oder ästhetische Einbussen durch sichtbare Metallklammern bei Teilprothesenlösungen entfallen.

Dentale Implantate und darauf befestigte Rekonstruktionen haben hohe Erfolgs- und Überlebensraten von rund 90-95\% nach 10 Jahren [2, 3]. Allerdings hängt der langfristige Erfolg der Behandlung von verschiedenen Parametern, wie allgemeinmedizinischem Gesundheitszustand des Patienten, korrekter Indikationsstellung, chirurgischer und prothetischer Umsetzung und nicht zuletzt Mundhygiene des Patienten sowie zahnärztlicher Nachsorge ab.

\section{Voraussetzung für Erfolg: Korrekte Implantatposition}

Ein entscheidender Erfolgsfaktor ist die korrekte Positionierung des Implantates. Einerseits muss das Implantat so in den Kieferknochen gesetzt werden, dass es einheilen kann, andererseits muss seine Position eine ästhetisch und funktionell korrekt gestaltete prothetische Versorgung erlauben. Wichtige anatomische Strukturen in der Umgebung, wie Nerven, Gefässe oder Wurzeln der Nachbarzähne dürfen dabei nicht Schaden nehmen. Zudem besteht bei inkorrekter Positionierung das Risiko für ästhetische Komplikationen wie Schleimhautrezessionen, die nur sehr schwer, wenn überhaupt, korrigiert werden können [4] [5] [Abb 1.]. Oft müssen in solchen Fällen Implantate entfernt werden, was einerseits invasiv und belastend ist, andererseits aufwändige Nachfolgebehandlungen verursacht, die trotz hohem Aufwand in manchen Fällen aufgrund der Vorschädigung erneut in kompromittierten Ergebnissen enden.

\section{Prothetische und chirurgische Planung}

Um Implanatfehlpositionen zu vermeiden, ist eine sorgfältige Planung und korrekte intraoperative Umsetzung nötig [Tab. 1]. Primär ist es wichtig, im Vorfeld mit dem Patienten zusammen das gewünschte prothetische Ziel (und allfällige Alternativen) zu definieren, z. B. im Falle einer Einzelzahnlücke eine implantatverankerte Krone, bei mehreren fehlenden Zähnen eine implantatverankerte Brücke oder eine implantatgetragene abnehmbare Prothese. Hierbei kann 
nach erfolgter Abrucknahme des Gebisses und Herstellung von Gipsmodellen der Zahntechniker das prothetische Ziel in Form von Wachszähnen simulieren und zusammen mit dem Zahnarzt auf seine Machbarkeit überprüfen. Die Simulation auf dem Gipsmodell kann mit dem Patienten besprochen werden, unter Umständen ein Kunststoffduplikat im Mund einprobiert werden.

Die Implantationsstelle wird dahingehend untersucht, ob die gewünschte Behandlung möglich ist. Dies schliesst eine klinische Untersuchung ein (Beurteilung der Lückenbreite, Knochenangebot, Schleimhautzustand), die in der Regel von einer Röntgenuntersuchung gefolgt ist, anhand derer die Knochendimensionen im zukünftigen Implantatbett vermessen werden. Wichtige Strukturen, wie Wurzeln der Nachbarzähne, Ausdehnung des Sinus maxillaris im Oberkieferseitenzahnbereich oder Verlauf des Canalis mandibularis mit dem entsprechenden Nerv werden dabei berücksichtigt. In der anschliessenden chirurgischen Implantatinsertion wird unter Lokalanästhesie nach Lappenbildung der Kieferknochen dargestellt und nach schrittweiser Osteotomie mit Bohrern aufsteigenden Durchmessers ein Bohrstollen präpariert. In diesen wird das schraubenförmige Implantat eingedreht. Weil der Knochen anschliessend an eine Zahnextraktion teilweise atrophiert, ist es oft notwendig, fehlendes Knochenvolumen mittels GBR Verfahren (Guided bone regeneration) zu regenerieren, was meist simultan in der gleichen operativen Sitzung nach der Insertion des Implantates erfolgen kann. Hierbei kommen heutzutage meist Materialien xenogenen Ursprungs zum Einsatz, bestehend aus einem granulatähnlichen Knochenersatzmaterial und einer langsam resorbierenden Kollagenmembran, wobei eine Vielzahl verschiedener Produkte und Materialien verfügbar sind.

Nach einer mehrmonatigen Heilungsphase kann dann, allenfalls nach zusätzlichem Schleimhautaufbau zwecks Volumenkompensation des atrophierten Gewebes, ein Abdruck inklusive Registrierung der Implantatposition genommen werden und nach entsprechenden Einproben dann die vom Zahntechniker hergestellte definitive Krone, Brücke oder Prothese auf dem Implantat oder den Implantaten eingegliedert werden.

Das beschriebene Verfahren wird, je nach klinischer Situation modifiziert, seit mehr als drei Jahrzehnten erfolgreich angewandt, beinhaltet jedoch gewisse Nachteile. Einerseits bietet die radiologische Abklärung nur ein zweidimensionales Bild. Die ebenfalls wichtige dritte Dimension, die der Kieferkammbreite entspricht, kann präoperativ relativ unzuverlässig beurteilt werden, zudem treten im konventionellen Röntgen Überlagerungen von Strukturen auf. Zudem erfolgt die Implantatbettpräparation und Implantatpositionierung freihändig und ist massgebend von der Erfahrung und vom Geschick des Operateurs abhängig.

\section{3-D Radiologie und computer-unterstützte Implantionsplanung}

Um diese Nachteile zu kompensieren, wurden in den letzten Jahren zur präoperativen Bildgebung vermehrt digitale Volumentomogramme (DVT) eingesetzt, die eine Röntgenanalyse in drei Dimensionen erlauben. Hierbei handelt es sich um eine Modifikation der 
Computertomographietechnik, bei der ein Strahlenkegel produziert wird und wodurch das Aufnahmeverfahren bezüglich Zeitaufwand und Strahlenbelastung verbessert wird [6]. Digitale Volumentomographen sind kleiner als bisherige medizinische CT-Geräte und erlauben dem Patienten, aufrecht zu sitzen, ohne ihn vollständig zu umschliessen [Abb. 2]. Um die resultierenden digitalen Röntgendaten optimal zu nutzen, wurde die diagnostische Betrachtungs-Software von verschiedenen Firmen weiterentwickelt und auf die Bedürfnisse in der Implantationsplanung angepasst. Hierbei ist einerseits eine Betrachtung der Schnittbilder in beliebiger Ebene möglich, aber auch die exakte Vermessung der Knochendimensionen, bis auf wenige zehntel Millimeter [7]. Aus einer Datenbank lassen sich verschiedene Implantattypen und -dimensionen auswählen und einblenden, im Sinne einer virtuellen Implantation [Abb. 3]. Allfällig fehlendes Knochenvolumen kann präoperativ erkannt werden und ein Knochenaufbau, je nach Ausmass des Knochendefekts vor oder gleichzeitig mit der Implantation, geplant werden. Dadurch kann sich der Operateur auf den bevorstehenden Eingriff besser vorbereiten und entsprechende Materialien (Implantate, Knochenersatzmaterialien) bestellen [8]. Aufgrund der besseren Vorhersehbarkeit des Eingriffs kann der Patient zuverlässiger aufgeklärt werden und über die anfallenden Kosten informiert werden.

Wie gesagt ist jedoch für eine korrekte Positionsplanung des Implantates die Kenntnis des zukünftigen prothetischen Zieles notwendig. Deshalb muss bei der virtuellen röntgenbasierten Planung die prothetische Information in der Software verfügbar sein. Ursprünglich wurde diese Information über eine Röntgenschablone, die der Patient bei der CT-Aufnahme im Mund hatte, einbezogen. Hierzu dublierte der Zahntechniker das ursprünglich in Wachs auf dem Gipsmodell geformte prothetische Ziel (Krone, Brücke, Prothese) in eine röntgendichte und somit im Röntgenbild sichtbare Kunststoffschablone, die als Röntgenschablone diente. Die in der Planungssoftware sichtbare weissliche Struktur des zukünftigen Zahnersatzes diente als prothetische Referenz. Dies bedingte jedoch, dass im Vorfeld die Röntgenschablone hergestellt werden musste, was kostenintensiv war und dass die Schablone während der Röntgen-Aufnahme korrekt positioniert war, ohne zu verrutschen. Bestehende DVT-Daten, die ev. kürzlich zu anderen Zwecken (ohne prothetische Röntgenschablone) angefertigt wurden, konnten für die Implantationsplanung nicht verwendet werden. Neuliche Entwicklungen erlauben es nun, dass Modelle der prothetischen Planung optisch eingescannt und somit digitalisiert werden und anschliessend in der Planungssoftware mit den DVT-Daten (ohne Röntgenschablone) über die Oberfläche bestehender Zahnstrukturen oder der festen Schleimhautanteile überlagert werden [Abb. 3]. Somit steht für die virtuelle Planung nun wiederum die prothetische Information zur Verfügung.

\section{Umsetzung der geplanten Implantatposition in eine Bohrschablone}

Damit die geplante Implantatposition nun auch in den Mund des Patienten übertragen werden kann, besteht der nächste Schritt nun darin, dass die virtuelle Implantatposition von der Software in 
eine Bohrschablone übertragen wird, die der Chirurg intraoperativ zur Führung seiner Bohrinstrumente nutzen kann. Anfänglich war hierfür abermals der Zahntechniker notwendig, der mittels teuerer und aufwändiger Methode in einem Koordinatengerät, in dem das Zahnmodell zusammen mit der ursprünglich verwendeten Röntgenschablone eingespannt wurde, die Position der Bohrhülse gemäss von der Software ermittelten Koordinaten umsetzen musste und die Bohrhülse manuell einklebte. Somit wurde die Röntgenschablone zur Bohrschablone. Neben relativ hohen Kosten war dieser Vorgang zeitaufwändig und fehleranfällig.

Auch hier ist es gelungen, den Prozess der Schablonenherstellung $\mathrm{zu}$ vereinfachen und $\mathrm{zu}$ optimieren. Basierend auf den digitalen Planungsdaten wird eine Bohrschablone halbautomatisch per CAD in der Software gestaltet und dann per CAM hergestellt. Schleifverfahren oder stereolithographische Verfahren werden zunehmend durch die günstigeren und einfacher verfügbaren 3D-Druckverfahren abgelöst. Beispielsweise hat der für diesen Zweck eingesetzte, am Zentrum für Zahnmedizin (ZZM) verfügbare Drucker eine hohe Druckauflösung von $12 \mu \mathrm{m}$ Schichtdicke und ist in der Lage rund 30 Schablonen gleichzeitig in einer Zeit von rund 3-4 Stunden zu drucken, was die Behandlungskosten gegenüber der manuellen zahntechnischen Herstellung senkt [Abb. 4].

\section{Schablonen-geführte Implantatchirurgie}

Die Schablone dient nun während der Operation zur Führung der Bohrinstrumente. Je nach Implantathersteller kommen unterschiedliche Instrumentenkombinationen zur Anwendung, jedoch erfolgt in der Regel die Führung der mit einem vertikalen Stopp versehenen Bohrer durch in der Schablone vorhandene Hülsen [Abb. 5]. Sowohl die in aufsteigendem Bohrerdurchmesser durchgeführte Bohrung als auch die Implantatinsertion selbst erfolgen durch die Schablone, was die Übertragung der geplanten Implantatposition gewährleistet. Dabei ist die Möglichkeit für ein seitliches Abgleiten oder eine Überinstrumentierung in der Länge limitiert. Speziell bei hartem Knochen, beim Bohren in eine schräge Knochenoberfläche oder in der Nähe von z. B. Nervenstrukturen bietet die Schiene Vorteile gegenüber freihändiger Instrumentierung, macht die Bohrung sicherer und einfacher.

\section{Genauigkeit der Positionsübertragung von der virtuellen in die reale Welt}

Allerdings kann aufgrund der nötigen Rotation der Bohrer durch die Führungsinstrumente eine gewisse Toleranz in der Führung nicht vermieden werden. Dies ist mit ein Grund, dass gegenüber der geplanten zur tatsächlich erreichten Implantatposition gemäss Studien Abweichungen von rund einem Millimeter auftreten können [9] [10]. Das Ausmass der möglichen Instrumentenauslenkung ist abhängig von der vom Operateur angewendeten Kraft und Richtung (er sollte die Instrumente möglichst passiv ohne Abwinkeln führen), andererseits von der Passgenauigkeit der Instrumente. Durch Modifikationen innerhalb der 3D-gedruckten Schablone konnte in einer in-vitro Studie die Instrumententoleranz signifikant reduziert werden [11]. Zudem konnte das Design der Schablonen 
dahingehend optimiert werden, dass die Schablone nicht mehr komplett die stützenden Zähne abdeckt und somit die Position der Schablone und der Bohrinstrumente visuell besser überprüft werden kann sowie die Wasserkühlung bei der Bohrung verbessert wird. Dennoch ist eine Resttoleranz zur Reduktion der Friktion zwischen den Komponenten und Vermeidung von Deformation oder Frakturen von Schienen notwendig. Die damit zusammenhängende leichte Abweichung der Implantatposition gegenüber dem virtuellen Plan scheint sich jedoch in der klinischen Anwendung nicht negativ auszuwirken, sofern in der Planung die empfohlenen Sicherheitsabstände zu kritischen anatomischen Strukturen von $2 \mathrm{~mm}$ eingehalten werden. Vorausgesetzt, dass genügend Knochen vorhanden ist, kann die Implantatinsertion auch nur durch eine kleine Öffnung der Schleimhaut mittels Stanze, ohne Aufklappung, erfolgen, was die postoperativen Beschwerden der Patienten deutlich reduziert [12].

\section{Komplikationen und Limitationen der Methode}

Klinische Studien haben gezeigt, dass die Implantatüberlebensrate von mittels CAIPP eingesetzter Implantate vergleichbar mit konventionell eingesetzten Implantaten ist [13]. Allerdings liegen relativ geringe Beobachtungszeiträume und eine im Vergleich geringere Implantatzahl vor. Es wurden aber auch Komplikationen und Einschränkungen beschrieben [10]. Zum einen wurden signifikante Abweichungen der Implantatposition berichtet, wenn Fabrikationsfehler, schlechter Sitz der Schablone auf den Zähnen bzw. Schleimhaut oder Verwindungen und Frakturen der Schienen aufgetreten sind und vom Behandler nicht bemerkt wurden. Weiter ist bei Patienten mit limitierter Mundöffnung unter Umständen der Einsatz der Instrumente aufgrund des vertikalen Platzbedarfs für das Einbringen der Bohrer in die Schablone nicht möglich. Auch Planungsfehler oder Fehlinterpretation der radiologischen Bilddaten können dazu führen, dass die radiologisch vermutete von der klinisch vorgefundenen Situation abweicht und somit Modifikationen des chirurgischen Protokolls, wie Knochenaugmentationen oder Änderungen der Implantatdimensionen oder -position, nach sich zieht.

\section{Indikationen, Vorteile und Nachteile}

Die Vorteile der CAIPP Technologie gegenüber der konventionellen Technik (bessere Operationsvorbereitung und Vorhersehbarkeit, Instrumentenführung, höhere Sicherheit) müssen den Nachteilen und Einschränkungen (höhere Strahlenbelastung, höhere Behandlungskosten und Anschaffungskosten für Hard- und Software, spezielles Chirurgie-Set, ev. Scanner und 3DDrucker) gegenübergestellt werden [Tab. 2]. Obwohl grundsätzlich jegliche Implantation mittels CAIPP erfolgen könnte, wurden klinische Situationen definiert, in denen der Einsatz dieser Technologie besonders indiziert ist [Tab. 3]. Grundsätzlich beinhaltet dies Situationen, in denen die konventionelle Röntgenbildgebung und klinische Untersuchung unzureichend sind und zusätzliche Abklärungen zum Vermeiden risikoreicher und invasiver Eingriffe fordern. Hinzu kommen Situationen, in denen die Instrumentenführung zur Reduzierung von manuellen Fehlern bei der 
Knochenbettaufbereitung gewünscht ist, wie Implantation in nicht ausgeheilte Zahnalveolen (Sofort- oder Frühimplantation nach Zahnextraktion) oder ungünstige Knochenverhältnisse (z.B. schräg verlaufender, kortikaler Knochen) sowie lappenfreier Operation mit beschränkter intraoperativer Orientierungsmöglichkeit (Abb. 6-9).

Durch die in Zukunft hoffentlich reduzierte Strahlenbelastung, sinkende Kosten der Geräte und Schablonen sowie zunehmende Vereinfachung der Abläufe und der Bedienung könnte sich der Indikationsbereich deutlich erweitern und die CAIPP Technologie im Rahmen der generell stattfindenden Digitalisierung in der Zahnmedizin zur Routineanwendung entwickeln. 


\section{Key messages}

- Dentale Implantate als Verankerungselemente für prothetische Rekonstruktionen stellen eine etablierte Behandlungsmethode mit guten Langzeitresultaten dar.

- Die Planung einer Implantation muss anatomische und prothetische Aspekte beinhalten und das Implantat muss an anatomisch und prothetisch korrekter Position stehen.

- Auf CT-/DVT-Röntgendaten basierende computer-assistierte Implantatplanung erlaubt eine umfassende 3-dimensionale präoperative Analyse und Entscheidungsfindung. Die auf der computer-unterstützten Planung beruhende, meist CAD-CAM gefertigte Bohrschablone hilft durch Instrumentenführung, die präoperativ festgelegte virtuelle Implantatposition intraoperativ zu übertragen.

\section{Lernfragen}

Eine präimplantologische Diagnostik und Planung beinhaltet immer (Mehrfachantworten möglich):

a) eine klinische Untersuchung

b) Bildgebung mittels Computertomographie oder digitaler Volumentomographie

c) Planung des prothetischen Zieles

d) anamnestische Erhebung des Patientenwunsches und der finanziellen Möglichkeiten (richtige Antworten a), c), d))

Die CAIPP Technologie trägt dazu bei, dass (Mehrfachantworten möglich):

a) die Implantatüberlebensrate steigt

b) der Operateur eventuelle Knochendefizite präoperativ besser voraussehen kann

C) die Behandlungskosten einer Implantatversorgung sinken

d) Patienten weniger invasiv behandelt werden können, sofern genügend Knochen an der Implantationsstelle vorhanden ist

(richtige Antworten b), d))

Speziell in folgenden Situationen bietet die CAIPP Technologie Vorteile (Mehrfachantworten möglich):

a) polymorbide Patienten mit hohem operativen Risiko

b) Patienten mit Einzelzahnlücken

c) Patienten mit anatomisch schwierigen Knochenverhältnissen

d) junge Patienten vor Abschluss des Knochenwachstums

(richtige Antworten a), c)) 


\section{Tabellen}

\begin{tabular}{|c|c|c|c|c|c|c|}
\hline & Befunderhebung & $\begin{array}{l}\text { Röntgen- } \\
\text { bildgebung }\end{array}$ & $\begin{array}{l}\text { Chirurgische } \\
\text { Planung }\end{array}$ & $\begin{array}{l}\text { Chirurgische } \\
\text { Schablone }\end{array}$ & Implantation & Prothetik \\
\hline Konventionell & $\begin{array}{l}\text { Anamnese, } \\
\text { klinische } \\
\text { Untersuchung }\end{array}$ & $\begin{array}{l}\text { Konventionelles } \\
\text { Röntgen mit } \\
\text { Röntgenschablone } \\
\text { (OPT, Einzelzahn- } \\
\text { Rx) }\end{array}$ & $\begin{array}{l}\text { Röntgenbild- } \\
\text { betrachter, } \\
\text { Gipsmodelle, } \\
\text { klinischer Befund }\end{array}$ & $\begin{array}{c}\text { Manuelle } \\
\text { Modifikation der } \\
\text { Röntgenschablone }\end{array}$ & $\begin{array}{l}\text { Freihändig, chirurg. } \\
\text { Schablone als } \\
\text { Orientierungshilfe } \\
\text { bezüglich } \\
\text { prothetischem Ziel }\end{array}$ & $\begin{array}{l}\text { Manuell oder CAD- } \\
\text { CAM gefertigte } \\
\text { Rekonstruktion }\end{array}$ \\
\hline $\begin{array}{l}\text { Computer- } \\
\text { unterstützt }\end{array}$ & $\begin{array}{l}\text { Anamnese, } \\
\text { klinische } \\
\text { Untersuchung }\end{array}$ & $\begin{array}{l}\text { 3D-Röntgen (DVT, } \\
\text { CT), je nach } \\
\text { CAIPP System } \\
\text { mit/ohne Röntgen- } \\
\text { schablone }\end{array}$ & $\begin{array}{l}\text { Computersoftware } \\
\text { mit digitalen } \\
\text { radiologischen } \\
\text { Daten und digitalen } \\
\text { Modelldaten }\end{array}$ & $\begin{array}{c}\text { CAD-CAM } \\
\text { Herstellung } \\
\text { Bohrschablone }\end{array}$ & $\begin{array}{l}\text { Geführt durch } \\
\text { Bohrschablone }\end{array}$ & $\begin{array}{l}\text { Manuell oder CAD- } \\
\text { CAM gefertigte } \\
\text { Rekonstruktion }\end{array}$ \\
\hline
\end{tabular}

Tabelle 1: Vereinfachter Ablauf einer konventionellen und computer-unterstützten, schablonengeführten implanatprothetischen Behandlung im Vergleich

\section{Vorteile:}

- Bessere Operationsvorbereitung und Vorhersehbarkeit der Anatomie dank 3D Bildgebung und virtueller Implantationsplanung

- Instrumentenführung bei der Operation, dadurch Reduktion von manuellen Fehlern, höhere Sicherheit und Genauigkeit

- Hohe Patientenakzeptanz und positiver Marketingeffekt

- Geringere postoperative Morbidität, wenn im Zusammenhang mit lappenfreier Operationstechnik eingesetzt

- Ev. Vorfertigung von prothetischen Komponenten basierend auf den digitalen Planungsdaten

\section{Nachteile:}

- Infrastrukturelle Anforderungen für CT/DVT-Scanner, Hard- und Software, ev. optischen Scanner, Chirurgie-Set

- Logistischer und zeitlicher Mehraufwand für DVT-Überweisung, Einscannen von Modellen, 3D Diagnostik und virtuelle Planung, Bestellung von Schablonen, speziellen Bohrern und Implantaten unter Berücksichtigung von Lieferfristen

- Relativ häufig intraoperative Anpassungen/Korrekturen nötig gegenüber der Planung

- Höhere Strahlenbelastung

- Höhere Kosten, v.a. durch 3D-Röntgen und Schablonenherstellung

Tabelle 2: Vor- und Nachteile der computer-assistierten, schablonen-geführten Implantation

\section{Indikationen für CAIPP Technologie:}

- Gewünschte 3D Bildgebung in Fällen mit komplexen anatomischen Verhältnissen (z.B. nach Trauma, resektiver Therapie, Spaltpatienten etc.)

- Basierend auf klinischer und konventionell-radiologischer Untersuchung vermutetes ungenügendes Knochenangebot

- Optimierung der Implantatposition in Fällen mit unvorteilhafter Knochenmorphologie (Bohrerführung)

- Minimal-invasive Chirurgie (lappenfreie Operation, z.B. bei antikoagulierten Patienten).

- Vorfabrikation prothetischer Komponenten bei Sofortversorgung 
Tabelle 3: Indikationen für computer-assistierte, schablonen-geführte Implantation (modifiziert nach [14]) 


\section{Abbildungslegenden}

Abbildung 1: Fehlpositioniertes Implantat in Regio des rechten oberen mittleren Schneidezahnes und daraus resultierende Schleimhautrezession. Teile des Titianimplantates sind exponiert, ein Teil wurde behelfsweise mittels rosa Keramik kompensiert. Die Rezession hat zu einem ästhetischen Misserfolg geführt, und aufgrund erschwerter Reinigbarkeit haben Bakterienbeläge zu einer peri-implantären Entzündung geführt. Eine Explantation und erneute, aufwändige Therapie inkl. Defektrekonstruktion ist für ein zufriedenstellendes ästhetisches Resultat unumgänglich.

Abbildung 2: Patient bei einer Aufnahme in einem digitalen Volumentomographiegerät. Die einander gegenüberliegende Röhre und Detektor kreisen um den Patienten. Eine Aufnahme dauert je nach Gerät und Einstellungen ca. 15-30 Sekunden.

Abbildung 3: Bildschirmfoto aus einer virtuellen Planung. Links oben die 3D-Rekonstruktion mit überlagertem Zahnmodell des prothetischen Zieles (violette Zahnstrukturen). In den anderen drei Feldern Ansichten in Schnittbildern. Violette Konturlinie mit sichtbarem prothetischem Ziel (eine Implantat-Krone im Oberkiefer rechts, zwei Implantat-Kronen im Oberkiefer links). Rosarot virtuell positionierte Implantate, im Alveolarknochen an prothetisch korrekter Position stehend, unter Umgehung einer Perforation des Sinus maxillaris links (sagittales Schnittbild oben rechts).

Abbildung 4: CAD-CAM hergestellte Schablone aus dem 3D-Drucker, aufliegend auf der Restbezahnung. Integrierte Führungshülsen an den geplanten Implantationsstellen (zwei Implantate im Frontbereich, eines im Seitenzahnbereich).

Abbildung 5: Schablonengeführte Bohrung in den freigelegten, sehr schmalen Alveolarknochen im Unterkiefer rechts.

Abbildungen 6-9: Beispiel einer lappenfreien, schablonengeführten Implantation unmittelbar nach Zahnextraktion bei einem betagten, polymorbiden Patienten. Der seitliche obere Schneidezahn wird aufgrund einer Kronenfraktur entfernt.

Abbildung 7: Implantatbettpräparation durch schablonengeführte Bohrung, ohne Lappenbildung.

Abbildung 8: Situation unmittelbar vor Implantatinsertion.

Abbildung 9: Implanatgetragene Krone nach der Montage. 


\section{Bibliografie}

1. Strassburger C, Kerschbaum T, Heydecke G. Influence of implant and conventional prostheses on satisfaction and quality of life: A literature review. Part 2: Qualitative analysis and evaluation of the studies. The International journal of prosthodontics. 2006;19(4):339-48.

2. Lekholm U, Gunne J, Henry PJ, Higuchi K. Survival of the Brånemark Implant in Partially Edentulous Jaws: A 10-Year Prospective Multicenter Study. 1999.

3. Moraschini V, Poubel LA, Ferreira VF, Barboza Edos S. Evaluation of survival and success rates of dental implants reported in longitudinal studies with a follow-up period of at least 10 years: a systematic review. International journal of oral and maxillofacial surgery. 2015;44(3):377-88.

4. Evans CD, Chen ST. Esthetic outcomes of immediate implant placements. Clinical oral implants research. 2008;19(1):73-80.

5. Burkhardt R, Joss A, Lang NP. Soft tissue dehiscence coverage around endosseous implants: a prospective cohort study. Clinical oral implants research. 2008;19(5):451-7.

6. Harris D, Horner K, Grondahl K, Jacobs R, Helmrot E, Benic GI, et al. E.A.O. guidelines for the use of diagnostic imaging in implant dentistry 2011. A consensus workshop organized by the European Association for Osseointegration at the Medical University of Warsaw. Clin Oral Implants Res. 2012;23(11):1243-53.

7. Loubele M, Guerrero ME, Jacobs R, Suetens P, van Steenberghe D. A comparison of jaw dimensions and quality asessments of bone characteristics with cone-beam CT, spiral tomography, and multi-slice spiral CT. Int J Oral Maxillofac Implants. 2007;22:446-54.

8. Schneider D, Sancho-Puchades M, Hämmerle CHF, Jung RE. Comparison of conventional and computer-assisted implant planning and placement in partially edentulous patients. completed.

9. Van Assche N, Vercruyssen M, Coucke W, Teughels W, Jacobs R, Quirynen M. Accuracy of computer-aided implant placement. Clin Oral Implants Res. 2012;23 Suppl 6:112-23.

10. Schneider D, Marquardt P, Zwahlen M, Jung RE. A systematic review on the accuracy and the clinical outcome of computer-guided template-based implant dentistry. Clin Oral Implants Res. 2009;20 Suppl 4:73-86.

11. Schneider D, Schober F, Grohmann P, Hammerle CH, Jung RE. In-vitro evaluation of the tolerance of surgical instruments in templates for computer-assisted guided implantology produced by 3-D printing. Clin Oral Implants Res. 2014.

12. Nkenke E, Eitner S, Radespiel-Tröger M, Vairaktaris E, Neukam FW, Fenner M. Patientcentred outcomes comparing transmucosal implant placement with an open approach in the maxilla: a prospective, non-randomized pilot study. Clin Oral Implants Res. 2007;18(2):197-203.

13. Hultin M, Svensson KG, Trulsson M. Clinical advantages of computer-guided implant placement: a systematic review. Clin Oral Implants Res. 2012;23 Suppl 6:124-35.

14. Hämmerle $\mathrm{CH}$, Stone $\mathrm{P}$, Jung RE, Kapos T, Brodala N. Consensus statements and recommended clinical procedures regarding computer-assisted implant dentistry. Int J Oral Maxillofac Implants. 2009;24 Suppl:126-31. 


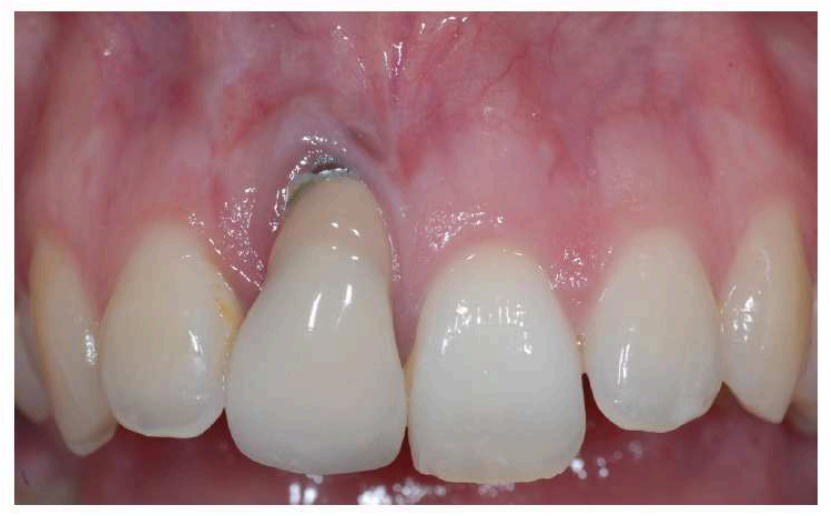

Figure 1

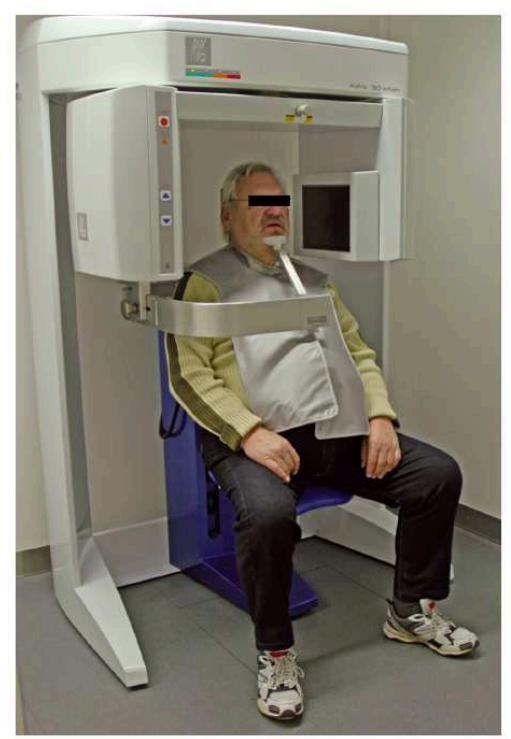

Figure 2

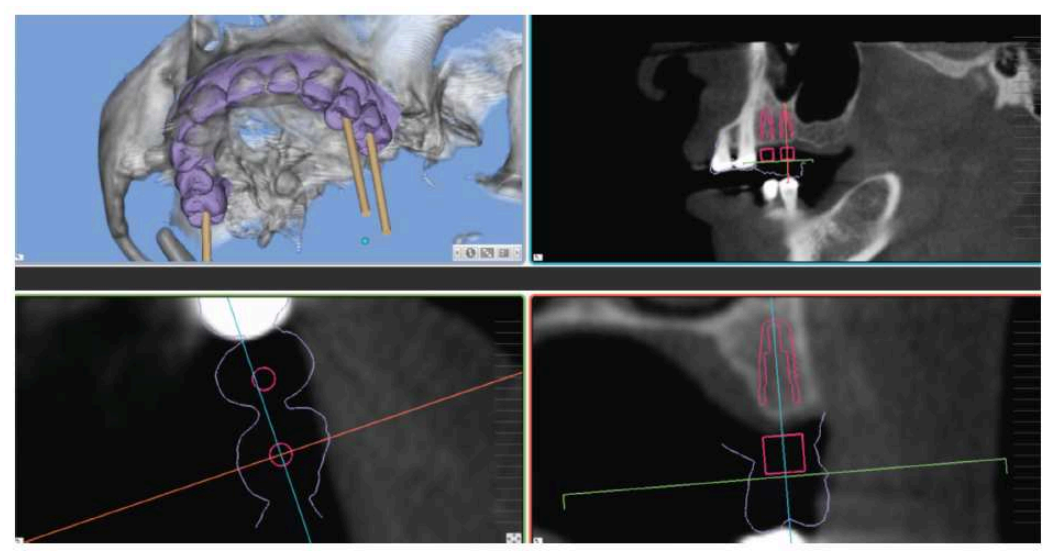

Figure 3 


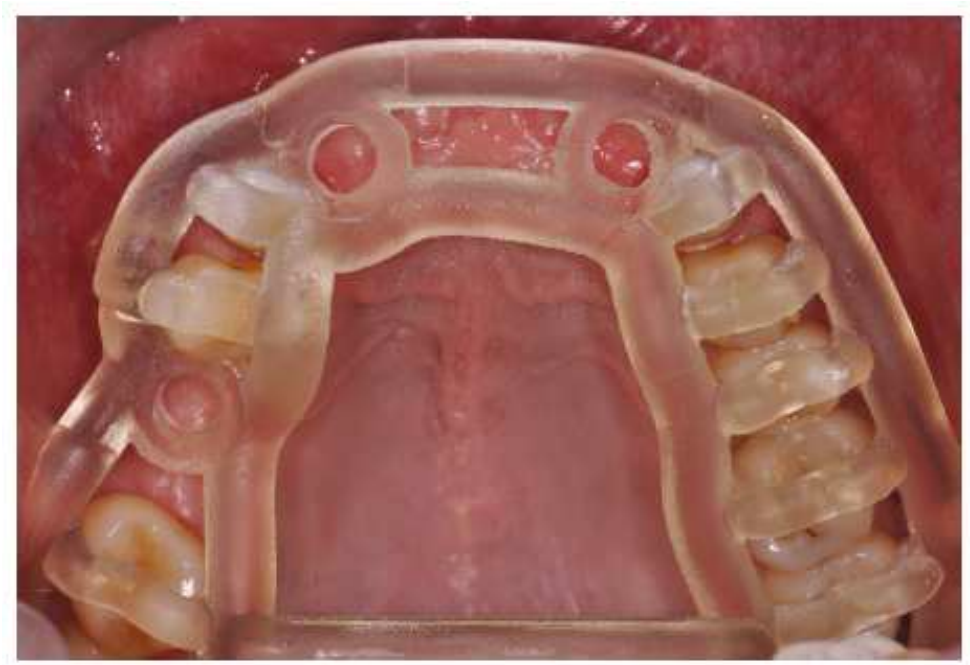

Figure 4

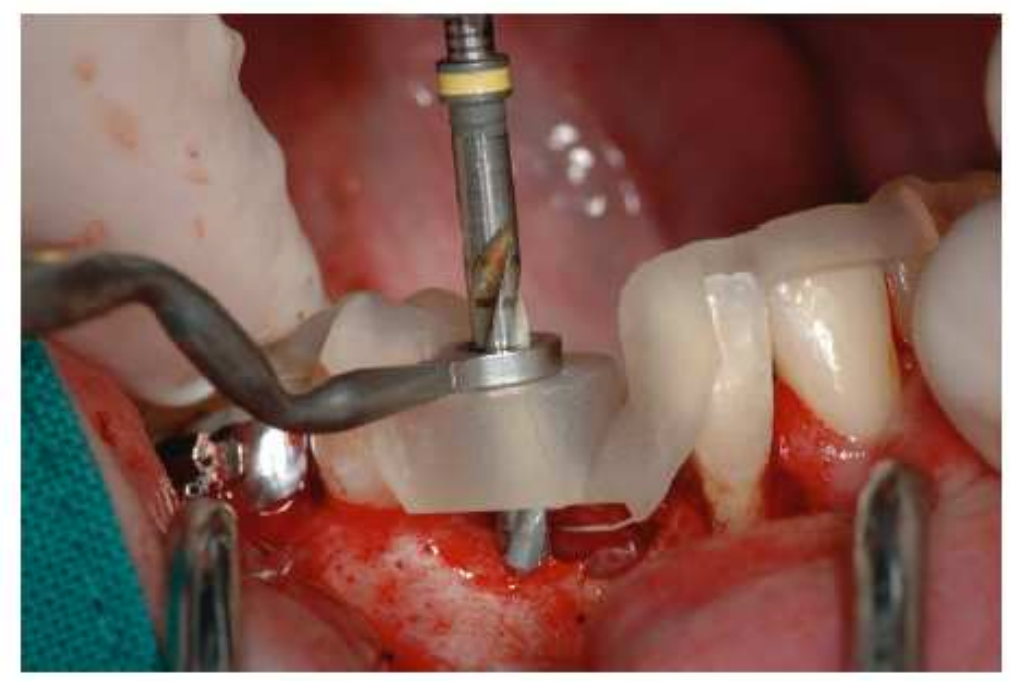

Figure 5

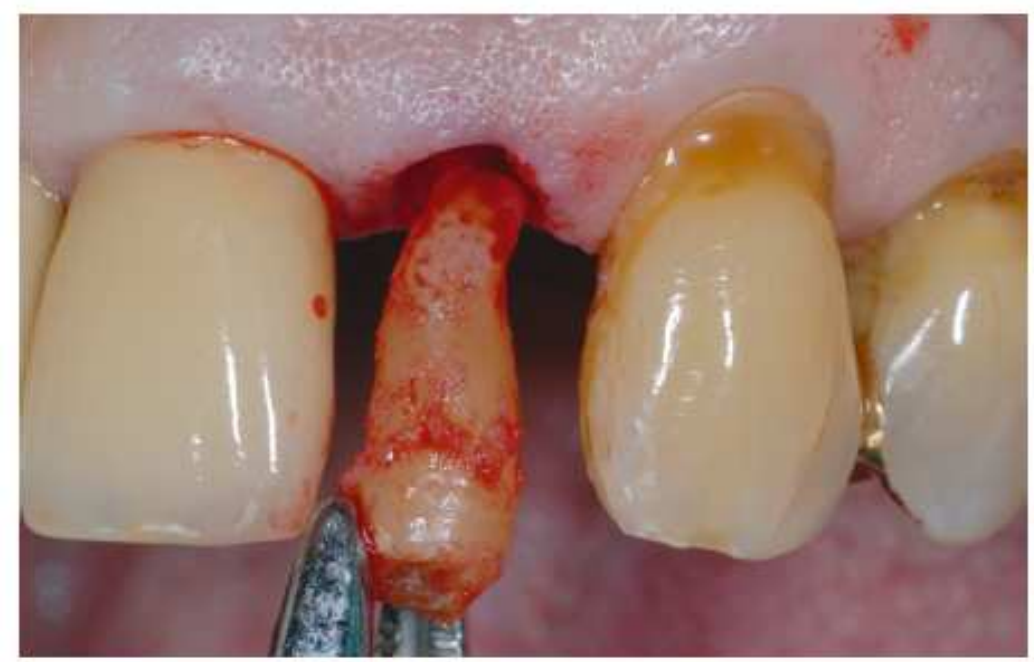

Figure 6 


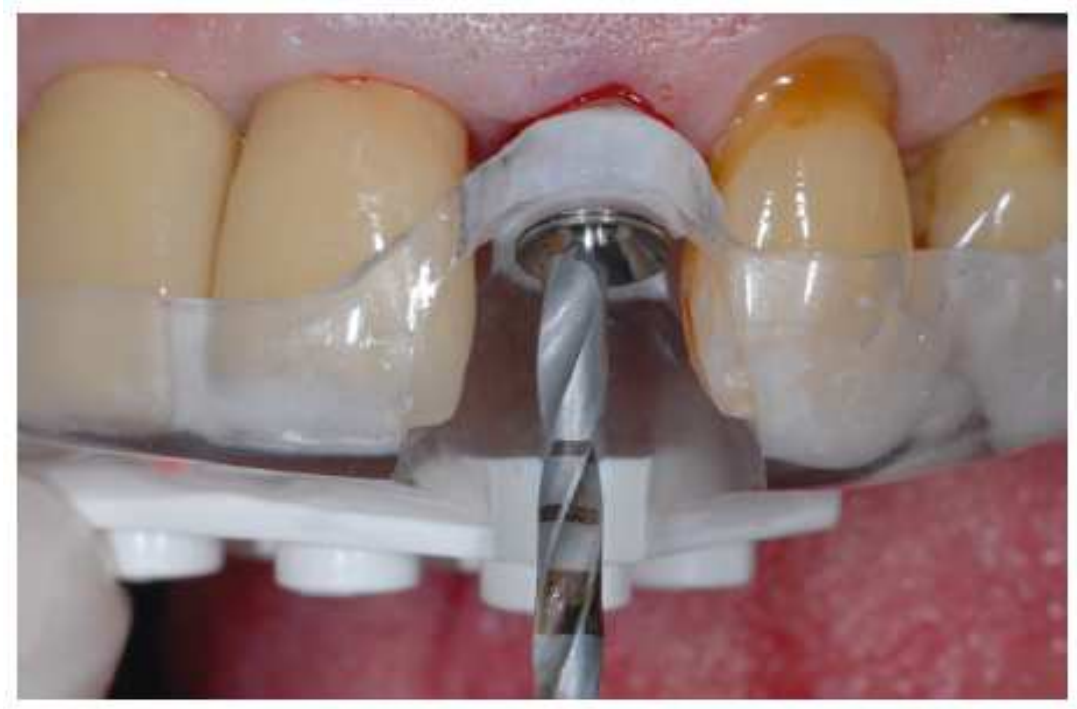

Figure 7

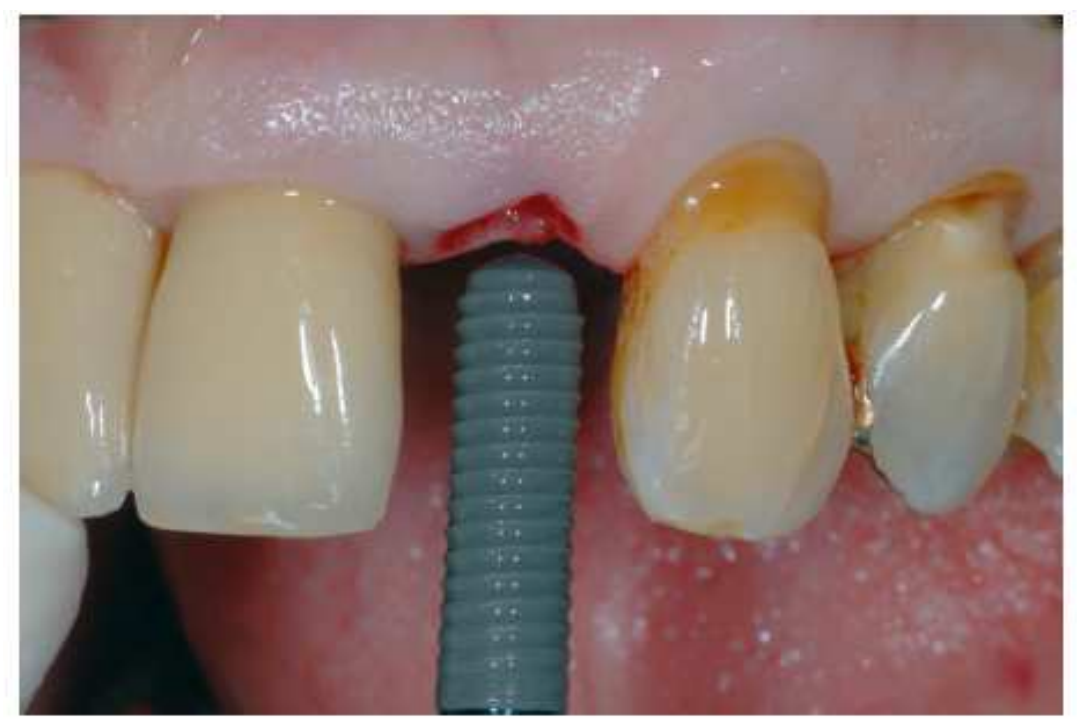

Figure 8

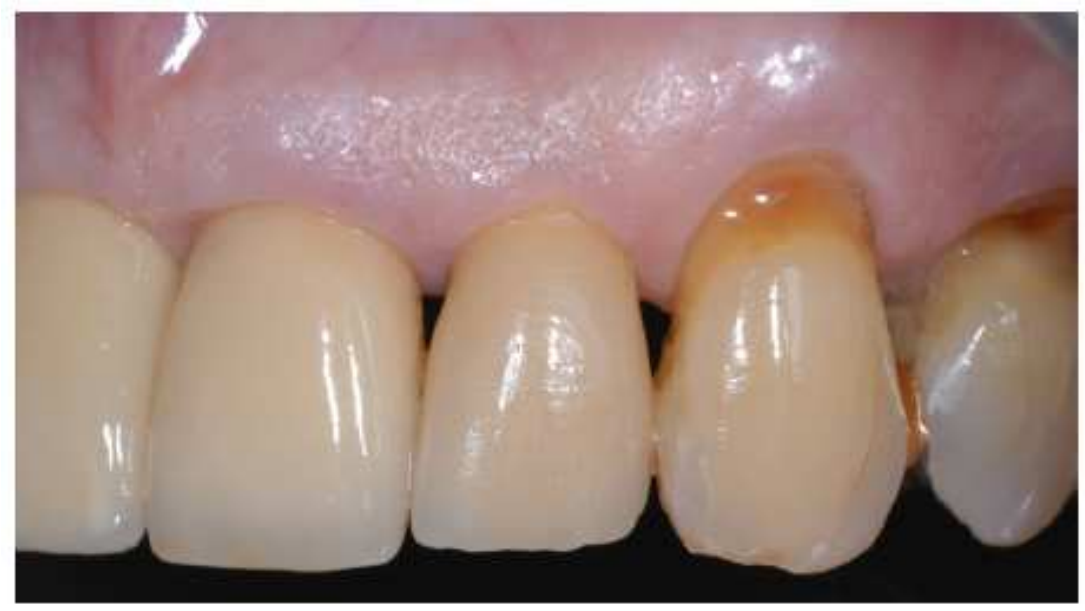

Figure 9 